\title{
Phase diagram of a chiral random matrix model with $2+1$ flavors
}

\author{
H. Fujii ${ }^{* \dagger}$ \\ Institute of Physics, University of Tokyo, Tokyo 153-8902, Japan \\ E-mail: hfujiilphys.c.u-tokyo.ac.jp \\ T. Sano \\ Department of Physics, University of Tokyo, Tokyo 113-0033, Japan \\ Institute of Physics, University of Tokyo, Tokyo 153-8902, Japan \\ E-mail: tsano@nt1.c.u-tokyo.ac.jp
}

\begin{abstract}
We recently proposed a chiral random matrix model incorporating the $\mathrm{U}_{\mathrm{A}}(1)$ breaking determinant term that brings about a flavor-number dependence of the chiral phase transition. We apply this model here for study of the phase diagram with degenerate up, down quark mass $m_{\mathrm{ud}}$ and the strange quark mass $m_{\mathrm{s}}$ at finite temperature and quark chemical potential. With zero chemical potential the model shows a first-order phase transition at finite temperature for smaller quark masses than a critical curve in the $m_{\mathrm{ud}}-m_{\mathrm{s}}$ plane, and this first-order region is enlarged in the $m_{\mathrm{ud}^{-}}{ }^{-}$ $m_{\mathrm{s}}$ plane when the chemical potential becomes nonzero, which is in accord with the expectation for the QCD critical point in the QCD phase diagram.
\end{abstract}

The XXVII International Symposium on Lattice Field Theory - LAT2009

July 26-31 2009

Peking University, Beijing, China

\footnotetext{
* Speaker.

†Work supported in part by Grant-in-Aid of MEXT, Japan (No. 19540269 and 19540273).
} 


\section{Introduction}

The chiral random matrix (ChRM) model [1] is defined with the fermion determinant in zero space-time dimension and may be regarded as a schematic model for study of the QCD thermodynamics. It was applied extensively in Ref. [2] to explore its phase diagram at finite temperature $T$ and quark chemical potential $\mu$, which revealed the phase structure with a tri-critical point (TCP) on the chiral phase boundary in the $T-\mu$ plane in the chiral limit, in accord with the structure obtained in other models for QCD [3]. In reality, the nonzero quark mass $m_{\mathrm{ud}}$ smears out the second-order boundary line and the TCP turns to be a simple critical point appearing at the end of the first-order line [4. 5]. This QCD critical point (QCD-CP) has attracted much attention from the viewpoint of the possible experimental confirmation as a critical evidence for the QCD phase transition [3, 可].

The phase structure is sensitive to the value of the strange quark mass $m_{\mathrm{s}}$. For $m_{\mathrm{s}}$ less than a certain critical value with the vanishing up-down quark mass $m_{\mathrm{ud}}=0$, the chiral transition at finite $T$ and at zero $\mu$ is expected to be of the first order [7]. In this case, there would be no TCP in the $T-\mu$ plane, provided that the finite $\mu$ makes the transition stronger. This qualitative feature should be retained even in the mean-field models. On the contrary, however, the ChRM model used in Ref. [2] has no flavor-number dependence in its phase structure, and it is favorably interpreted as a model with two flavors since it predicts a second-order transition at finite temperature and with zero chemical potential.

We recently proposed a ChRM model which incorporates the $\mathrm{U}_{\mathrm{A}}(1)$ breaking determinant term by considering a distribution for the number of the zero modes [8]. Owing to this term, the finitetemperature transition of the model becomes of the first order for three massless flavors whereas it remains of the second order for two flavors. In this paper, we explore the phase diagram of the ChRM model which has the flavor number dependence through the $\mathrm{U}_{\mathrm{A}}(1)$ breaking determinant term [8, 9]. This is the first study of the phase diagram in the space of $T, \mu, m_{\mathrm{ud}}$ and $m_{\mathrm{s}}$ in the ChRM model.

\section{A random matrix model with the determinant interaction}

Dynamical breaking of chiral symmetry in the quark system may be signaled by the nonvanishing density of the Dirac eigenvalues at the origin $\rho(0) \neq 0$, through the Banks-Casher relation $\langle\bar{q} q\rangle \propto \rho(0) / V$ with $V$ being the space-time volume. These low-lying eigenvalues are thought to originate from the localized instantons and other non-perturbative gauge field effects. In the ChRM model, focusing on the properties $\rho(\lambda)$ at small $\lambda$, we restrict the Dirac operator into the space of the small-eigenvalue modes. We may divide intuitively these quasi-zero modes into two categories: $N_{+}+N_{-}$topological zero modes associated to $N_{+}$instantons and $N_{-}$anti-instantons, and $2 N$ nearzero modes stemming from other dynamical effects [10, 8]. The numbers of these modes, $N_{+}, N_{-}$ and $2 N$, are considered to be proportional to the space-time volume $V$. Then the Dirac operator of the model takes the form

$$
D=\left(\begin{array}{cc}
0 & i R+C \\
i R^{\dagger}+C^{T} & 0
\end{array}\right),
$$

where the block structure is required by chiral symmetry. The complex matrix $R \in \mathbf{C}^{\left(N+N_{+}\right) \times\left(N+N_{-}\right)}$ is treated as random variable reflecting the non-perturbative gauge field dynamics. The matrix $C$ 
represents the effects of finite temperature $T$ and chemical potential $\mu$, and is chosen to be in a simple form [2]

$$
C=\left(\begin{array}{ccc}
(\mu+i T) \mathbf{1}_{N / 2} & 0 & 0 \\
0 & (\mu-i T) \mathbf{1}_{N / 2} & 0 \\
0 & 0 & 0
\end{array}\right)
$$

The temperature $T$ appears as a deterministic part to $D$, while $\mu$ breaks its anti-hermiticity leaving $D$ invariant under $\mu \leftrightarrow-\mu$. Note that the topological zero mode sector of $C$, i.e., $N_{+} \times N_{-}$bottomright block, is set to zero without any effect of $T$ or $\mu$. This may be interpreted as the fact that the localized instanton zero mode is insensitive to the boundary condition set by the temperature and the chemical potential in the Matsubara formalism. The partition function of the Gaussian ChRM model for fixed $N_{+}$and $N_{-}$is defined with this Dirac operator as:

$$
Z_{N_{+}, N_{-}}=\int d R e^{-N \Sigma^{2} \operatorname{tr} R R^{\dagger}} \prod_{f=1}^{N_{f}} \operatorname{det}\left(D+m_{f}\right) .
$$

Note that the partition function for $N_{+}=N_{-}=0$ reduces to the one used in [2].

Within a finite space-time volume, the numbers of the instantons and anti-instantons are fluctuating, and accordingly one should sum up the partition function $Z_{N_{+}, N_{-}}$over $\left(N_{+}, N_{-}\right)$with a certain distribution,

$$
Z^{\mathrm{RM}}=\sum_{N_{+}, N_{-}} P\left(N_{+}\right) P\left(N_{-}\right) Z_{N_{+}, N_{-}},
$$

where we have assumed independent distributions $P\left(N_{ \pm}\right)$for $N_{ \pm}$. Consequently, the total number of the quasi-zero modes, $2 N+N_{+}+N_{-}$, becomes a varying quantity. For $P\left(N_{ \pm}\right)$the Poisson distribution seems reasonable at a glance, but it leads to an unbound effectivel potential. In order to avoid this difficulty we use the binomial distribution instead [8]:

$$
P\left(N_{ \pm}\right)=\left(\begin{array}{c}
\gamma N \\
N_{ \pm}
\end{array}\right) p^{N_{ \pm}}(1-p)^{\gamma N-N_{ \pm}},
$$

where $\gamma N$ is the maximum value of $N_{ \pm}$with $\gamma$ being $\mathscr{O}(1)$, and the $p$ may be interpreted as the probability for one topological zero mode to appear.

After the bosonization with introducting an auxiliary variable $S^{f g} \sim q_{R}^{\dagger f} q_{L}^{g}$, which yields the order parameter martix, we find the complete partition function as

$$
Z^{\mathrm{RM}}(\mathscr{M}, T, \mu)=\int d S e^{-2 N \Omega(S ; \mathscr{M}, T, \mu)}
$$

with

$$
\begin{gathered}
\Omega=\frac{1}{2} \Sigma^{2} \operatorname{tr} S^{\dagger} S-\frac{1}{4} \ln \operatorname{det}\left[(S+\mathscr{M})\left(S^{\dagger}+\mathscr{M}^{\dagger}\right)-(\mu+i T)^{2}\right]\left[(S+\mathscr{M})\left(S^{\dagger}+\mathscr{M}^{\dagger}\right)-(\mu-i T)^{2}\right] \\
-\frac{1}{2} \gamma\left[\ln (\alpha \operatorname{det}(S+\mathscr{M})+1)+\ln \left(\alpha \operatorname{det}\left(S^{\dagger}+\mathscr{M}^{\dagger}\right)+1\right)\right],
\end{gathered}
$$

where $\mathscr{M}$ is the mass matrix in the flavor space. This is the ChRM model with the $\mathrm{U}_{\mathrm{A}}(1)$ breaking determinant interaction. 

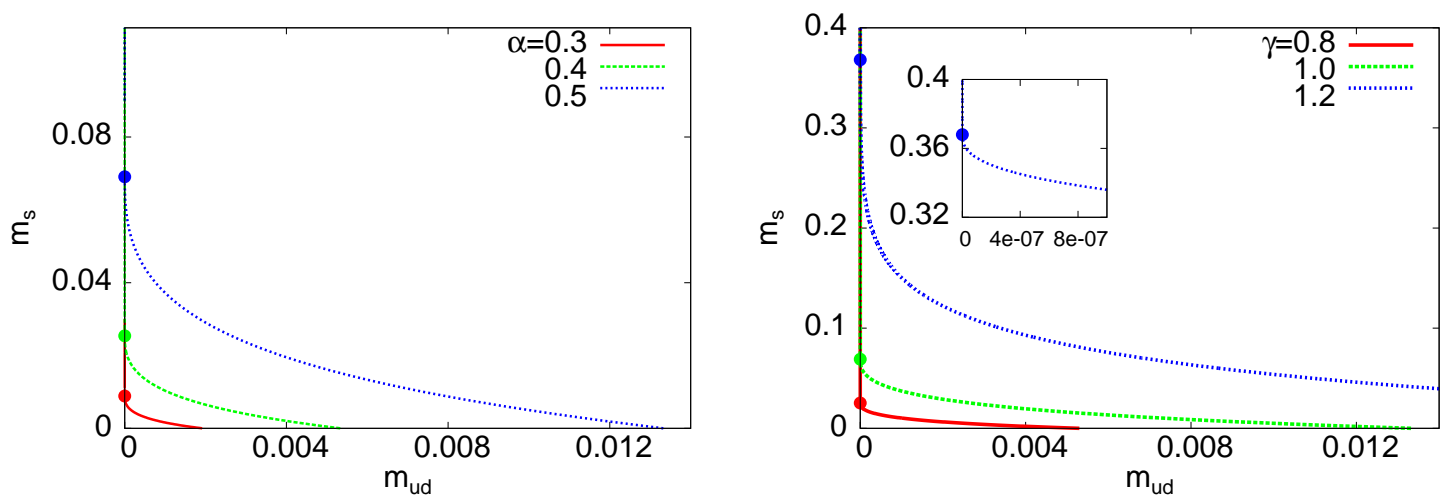

Figure 1: The secon-order phase transition lines on $m_{\mathrm{ud}}-m_{\mathrm{s}}$ plane for several values of $\alpha$ with $\gamma=1$ (left) and for several values of $\gamma$ with $\alpha=0.5$ (right). The TCP for each parameter is denoted by a dot.

Fluctuation properties of this model at finite temperatures for the cases with two and three equal-mass flavors are reported in Ref. [8]. In this paper, we examine the phase structure in the $2+1$ flavor case $\mathscr{M}=\operatorname{diag}\left(m_{\text {ud }}, m_{\text {ud }}, m_{\mathrm{s}}\right)$. Choosing $S=\operatorname{diag}\left(\phi_{\text {ud }}, \phi_{\text {ud }}, \phi_{\mathrm{s}}\right)$ we fix the ground state by the saddle point equations for $\phi_{\mathrm{ud}}$ and $\phi_{\mathrm{s}}$,

$$
\frac{\partial \Omega}{\partial \phi_{\mathrm{ud}}}=0, \quad \frac{\partial \Omega}{\partial \phi_{\mathrm{s}}}=0
$$

which becomes exact in the thermodynamic limit $N \rightarrow \infty$. We set $\Sigma=1$ since $\Sigma$ can be absorbed in $S$ by redefinition of other parameters, and leave $\alpha$ and $\gamma$ as free prameters.

\section{The phase diagram}

\subsection{Finite temperature $T \neq 0$ and zero chemical potential $\mu=0$}

It is generally expected that the chiral phase transition at finite temperature is the second order for $N_{f}=2$ massless quarks with $\mathrm{U}_{\mathrm{A}}(1)$ breaking, while it becomes the first order for $N_{f}=3$ massless quarks. This means that the order of the chiral phase transition $\left(m_{\mathrm{ud}}=0\right)$ for $N_{f}=2$ changes when we vary $m_{\mathrm{s}}$ as an additional external field. The point where the order of the transition changes is called the tri-critical (TCP) point.

Now that we have the first ChRM model which incorporates the determinant interaction term, we are ready to examine the effect of the quark masses on the chiral transition of this model in $m_{\mathrm{ud}}-$ $m_{\mathrm{s}}$ plane. In the left panel of Fig. 1, we draw in the $m_{\mathrm{ud}}-m_{\mathrm{s}}$ plane the critical lines below (above) which the finite temperature transition is the first order (cross-over), for $\alpha=0.3,0.4$ and 0.5 with $\gamma=1.0$. On the critical lines the system shows a second-order transition at a finite temperature. These lines approach the $m_{\mathrm{s}}$ axis as $m_{\mathrm{s}}^{\mathrm{TCP}}-m_{\mathrm{s}} \propto m_{\mathrm{ud}}^{2 / 5} \rightarrow 0$, as expected from the Landau-Ginzburg analysis. The endpoints $m_{\mathrm{s}}^{\mathrm{TCP}}$ are denoted with dots in Fig. 1. As we increse the weight $\alpha=$ $p /(1-p)$ of the topological zero mode, the first-order region is enlarged in $m_{\mathrm{ud}}-m_{\mathrm{s}}$ plane. As shown in the right panel of Fig. 1, the increase of the maximum number of the topological zero modes, $\gamma N$, also results in a wider first-order region. 


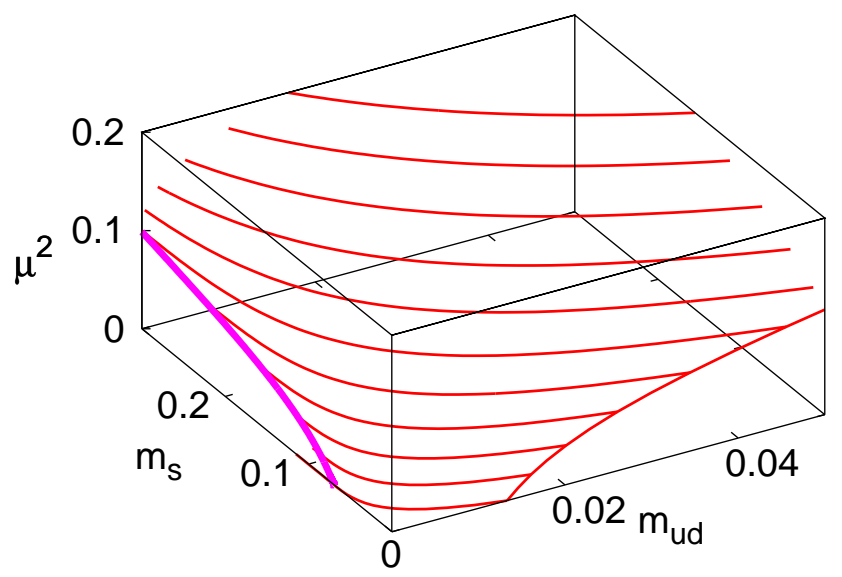

Figure 2: Critical surface in the $m_{\mathrm{ud}}-m_{\mathrm{s}}-\mu^{2}$ space with parameters $\gamma=1$ and $\alpha=0.5$. The bold line denotes the line of TCP.

\subsection{Finite temperature $T \neq 0$ and finite chemical potential $\mu \neq 0$}

Next we explore the phase structure of the model at finite chemical potential $\mu \neq 0$. In the earlier work of [2] using the model with no determinant term, it is found that the finite chemical potential $\mu$ makes the transition stronger, which generates the TCP in the $T-\mu$ plane in the chiral limit. Similar behavior is observed in the model with the determinant interaction. In Fig. 2] we plot a series of critical lines for several values of $\mu$, which as a whole spans a surface in the $m_{\mathrm{ud}}-m_{\mathrm{s}}-\mu^{2}$ space. The region of the first order transition becomes enlarged with increasing $\mu^{2}$. In the $m_{\mathrm{s}}-\mu^{2}$ plane, we obtain a line of TCP. This behavior is in accord with the standard scenario [3, 2, 11] suggesting the existence of the QCD-CP, provided that the transition is cross-over at $T \neq 0$ and $\mu=0$. We have checked by changing the values of $\alpha$ and $\gamma$ that this qualitative behavior is robust. The model parameters may depend on $\mu$ in general and it is possible to make the surface back-bend by varying $\alpha$ and/or $\gamma$ as functions of $\mu$, although their $\mu$-dependences are unknown within this model.

\section{Summary}

We have presented the first result on the phase diagram of the ChRM model in the space of $m_{\mathrm{ud}}-m_{\mathrm{s}}-\mu$. This is made possible with the ChRM model with the $\mathrm{U}_{\mathrm{A}}(1)$ breaking term. We have found taht the region of the first-order phase transition in the $m_{\mathrm{ud}}-m_{\mathrm{s}}$ plane increases with increasing anomaly parameters $\alpha$ and/or $\gamma$ at $\mu=0$. The nonzero quark chemical potential $\mu \neq 0$ makes the chiral phase transition stronger and the first-order transtion region is enlarged. This structure of the phase diagram in the space of $m_{\mathrm{ud}}-m_{\mathrm{s}}-\mu$ is robust in a wide range of the model parameters $\alpha$ and $\gamma$, and is in accord with the standard scenario for the QCD critical point. If one allows the parameters $\alpha$ or $\gamma$ to be $\mu$-dependent, it is possible to make the critical surface back-bend [12, 13]. 


\section{References}

[1] E. V. Shuryak and J. J. M. Verbaarschot, Nucl. Phys. A 560 (1993) 306; for review, J. J. M. Verbaarschot and T. Wettig, Ann. Rev. Nucl. Part. Sci. 50 (2000) 343.

[2] A. M. Halasz, A. D. Jackson, R. E. Shrock, M. A. Stephanov and J. J. M. Verbaarschot, Phys. Rev. D 58 (1998) 096007.

[3] For recent review, M. Stephanov, Proc. Sci., LAT2006 (2007) 024 [PoS (LAT20 06) 024 ].

[4] M. Asakawa and K. Yazaki, Nucl. Phys. A 504 (1989) 668.

[5] A. Barducci, R. Casalbuoni, S. De Curtis, R. Gatto and G. Pettini, Phys. Lett. B 231, 463 (1989); Phys. Rev. D 41, 1610 (1990).

[6] M. A. Stephanov, K. Rajagopal and E. V. Shuryak, Phys. Rev. Lett. 81, 4816 (1998).

[7] R. D. Pisarski and F. Wilczek, Phys. Rev. D 29 (1984), 338.

[8] T. Sano, H. Fujii and M. Ohtani, Phys. Rev. D 80 (2009) 034007; in these proceedings, POS (LAT2009) 222.

[9] H. Fujii and T. Sano, to be submitted.

[10] R. A. Janik, M. A. Nowak and I. Zahed, Phys. Lett. B 392 (1997) 155.

[11] F. Karsch, C. R. Allton, S. Ejiri, S. J. Hands, O. Kaczmarek, E. Laermann and C. Schmidt, Nucl. Phys. Proc. Suppl. 129, 614 (2004).

[12] P. de Forcrand and O. Philipsen, Nucl. Phys. B 673, 170 (2003).

[13] K. Fukushima, Phys. Rev. D 78, 114019 (2008); J. W. Chen, K. Fukushima, H. Kohyama, K. Ohnishi and U. Raha, Phys. Rev. D 80, 054012 (2009). 